\title{
Night-Vision Display Unlit during Uneventful Periods May Improve Traffic Safety
}

\author{
Rita Kovordányi, Torbjörn Alm, and Kjell Ohlsson
}

\begin{abstract}
It is always uncertain if a new assistance system will enhance traffic safety or not: empirical studies indicate that driving style may deteriorate when the driver experiences the increased safety margin created by an advanced driver assistance system. To minimize this negative effect on driving style, we redesigned a night vision system so that it appeared differently to the driver: we let the system's head-up display be turned off during operation, to be lit up only when the system detected an obstacle (e.g., a pedestrian or animal) on the road ahead. This presentation style was compared in a simulator study to the traditional solution of constantly lit-up display. The results indicate that drivers reacted more reliably (showed less variance in reaction times) using the new system, which implies that the lighting up of the IR-display constituted an effective warning. Also, drivers to a greater extent drove at normal (slower) speeds when using the re-designed system. More generally, systems offering discontinuous support (i.e. only in critical situations) may have less of a negative effect on driving style, as their presence is not felt as vividly by the driver.
\end{abstract}

Index Terms - night vision systems, advanced driver assistance systems, driving style, negative behavioral adaptation, re-design, discontinuous support

\section{INTRODUCTION}

$\mathrm{A}$ ssessment of the safety effect of newly developed advanced driver assistance systems (ADAS) is often based on engineering style mathematical calculations. For example, the safety effect of a night vision system (NVS) may be assessed on the basis of the number of reported accidents per year that occur during night-time driving and otherwise impaired visibility conditions (e.g., rain or fog). In addition, calculations could take into account how many cars in the future will use the newly developed NVS - the more cars using the system, the less probability for night-time collisions involving several vehicles. Moreover, the visibility of the road ahead might be estimated to increase by an additional $x$ meters

Rita Kovordányi is assistant professor in Cognitive Technology at the Department of Computer and Information Science, Linköpings Universitet, Linköping, SE-581 83 Sweden (phone: +46 13 281430; fax: +46 13 142231; e-mail: ritko@ida.liu.se).

Torbjörn Alm is head of the VR \& Simulation lab at the division of Industrial Ergonomics, Department of Mechanical Engineering, Linköpings Universitet, Linköping, SE-581 83 Sweden (e-mail: toral@ikp.liu.se).

Kjell Ohlsson is professor of Human-Machine Interaction at the division of Industrial Ergonomics, Department of Mechanical Engineering, Linköpings Universitet, Linköping, SE-581 83 Sweden (e-mail: kjeoh@ikp.liu.se). in vehicles using the newly developed NVS. Based on these figures, the safety effect of the newly developed NVS might be estimated to $y \%$ less accidents within the next $n$ years (see, e.g., [1] for similar estimates for Lane Departure Warning Systems).

Although these calculations are basically sound, they leave out an important factor: the driver. The driver may turn-off the system at periods, or may ignore the system's warnings when other priorities rule (e.g., getting to a meeting in time). Even more importantly, drivers may (partly unconsciously) adapt their driving style by driving faster and paying less attention to the traffic when supported by ADAS [2]. This phenomenon has been commonly termed negative behavioral adaptation in the empirical research literature [3]-[7]. In worst case, drivers may adapt their driving style to such an extent that the safety margin created by the driver assistance system is cancelled out [1], [8].

The point we want to make is that, instead of regarding the driver as a passive user of a technical system, the driver should be conceived of as the other half of an aggregate driving system, which consists of the driver in cooperation with the technical system [1]. Hence, any safety effects of a newly developed ADAS will depend on technical system performance in combination with driver behavior. For an ADAS to be a success, both these factors must work in the same direction.

\section{NIGHT VISION SYSTEMS}

Night vision systems make no exception from these problems. One evident problem is that drivers may tend to look too much on the night-vision display and pay too little attention to the actual road scene. Another, equally obvious problem is that drivers may be driving faster when visibility conditions improve as a result of the NVS.

NVS show an enhanced image of the road ahead based on one or two infrared sensors located in the front bumper of the car. Far infrared sensors are based on passive reception of infrared signals from the environment. These sensors result in better contrast between animate vs. inanimate objects, and is the system preferentially used for intelligent pedestrian warning detection, for example, by Honda [9].

Near infrared sensors actively emit infrared and microwave signals and receive the reflection of these from animate road users, as well as from vehicles and other colder objects. These systems provide a better overview of the road scene, as other 
vehicles, road signs and side-posts, and even road markings are included in the image.

The enhanced image is displayed on a head-up display mounted on the dashboard or is projected onto the windshield. Depending on the position of the display, drivers have to move their heads and eyes more or less from the road scene when looking at the display. Hence, refocusing of the eyes, both in the vertical plane and in depth, as well as refocusing of attention may be necessary.

The view angle of the image is often varied depending on driving speed (e.g. BMW, Honda), so that higher speeds result in a narrowing of the displayed image.

Today's top-of-the-line systems, such as Honda's intelligent NVS [10], use two sensors for stereo vision and distance estimation, and can intelligently interpret the road scene and warn the driver with auditory and visual alarm when pedestrians are detected on the road ahead (Fig. 1)

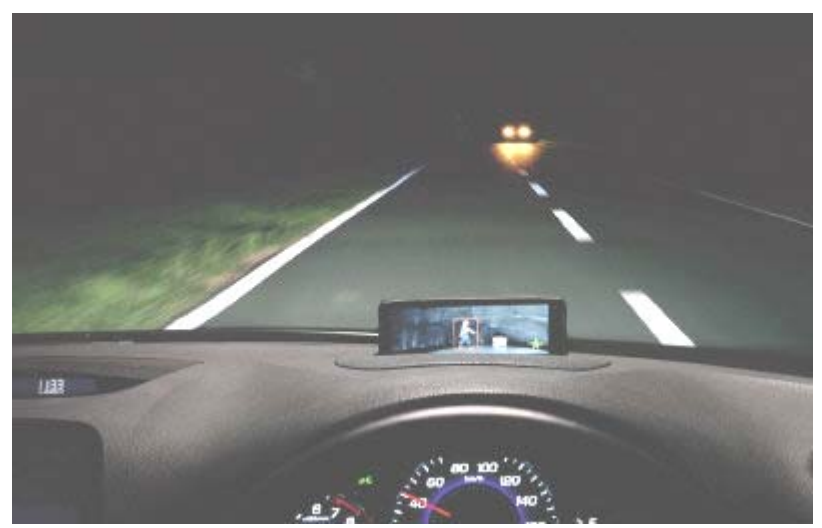

Fig. 1. NVS provide an enhanced view of the road ahead. The example shows Honda's intelligent NVS, which has detected a pedestrian. The system warns the driver by highlighting the pedestrian on the head-up display and by blinking an icon and sounding an alarm (more information can be obtained from Honda's web-site, [10])

\section{IMPACT ON DRIVING STYLE}

Negative behavioral adaptation is a widely observed phenomenon that also affects NVS [11]-[15]. One concern about these systems is that drivers may not detect peripheral obstacles that appear outside the view angle of the night vision display [16]. Also, drivers may look too often and too long on the head-up display instead of monitoring the road, which inevitably entails that drivers have to frequently refocus their attention when redirecting their gaze between the displayed image and the external road scene. Moreover, when drivers experience improved visibility conditions due to the nightvision display, they may want to drive faster [1].

\section{RATIONALE FOR A RE-DESIGN}

The singularly most important driving force to any change in driving style is an underlying change in perceptual input that is, a change in subjectively experienced visibility conditions and car maneuverability. So, when a night vision system improves subjectively experienced visibility of the road ahead, this will induce the driver to drive faster in order to maintain a constant (zero) risk level [2].

To mitigate this negative effect on driving style, we want to minimize the time when visibility condition improvements are experienced by the driver. In other words, we want, to prevent the driver from adapting to these improvements.

\section{THE RE-DESIGNED NIGHT VISION SYSTEM}

To keep the subsequent experimental comparison clear from confounds, we were careful not to introduce unnecessary changes to the original NVS. Hence, we focused on one dimension: how the driver would perceive improved visibility conditions. In other words:

1. The original system presented an enhanced view of the road ahead continuously during nighttime driving (Fig. 2).

2. The re-designed system's display was instead turned off during operation and was lit up only when the system detected an obstacle ahead. Note that lighting up the display also functioned as a visual warning to the driver. A sudden lighting up of the display not only evoked the driver's attention, but also informed the driver of the position and type of danger.

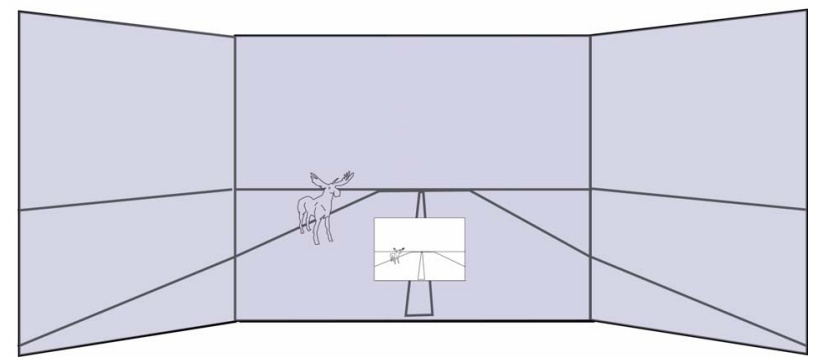

Fig. 2. The simulated night-vision display used in the experiment presented an enhanced view of the road scene overlaid the environment display. The display was continuously lit for the original system, but was unlit for the re-designed system during uneventful periods.

Both the original and the re-designed system worked in the same way in every other respect, besides the change in information presentation mentioned above. In particular, none of the systems provided auditory warnings to the driver.

The two NVSs used in the experiment were software-based. This meant, for example, that the systems could rely on faked sensor data obtained from the driving simulator. Hence, there was no need for an intelligent computer vision component in the system to detect pedestrians and animals (moose) that appeared on the road ahead of the driver. As the systems were software-based, system features, such as head-up display size and contrast, and required distance to obstacle when the redesigned system's display would be lit up, could be easily adjusted through the setting of software parameters [17]. 


\section{EVALUATION IN A DRIVING SIMULATOR EXPERIMENT}

We conducted a driving simulator study with the aim to evaluate the safety effects of the two NVSs. In particular, we wanted to see whether driving style and road monitoring patterns were affected less by the re-designed system as compared to the original NVS.

\section{A. Experimental design}

23 collage students ( 15 male and 8 female students), aged 20-38 years took part in the driving simulator experiment. All participants had had their driving license for at least two years.

The test consisted of $2 \times 30$-minute simulated nighttime driving on a $90 \mathrm{~km}$ speed limited highway, with sporadic oncoming traffic.

Participants completed two sessions, driving the same route, but using different NVSs, namely

1. using the original NVS

2. using the re-designed system with unlit display during uneventful periods

We employed a balanced experimental design, meaning that half the participants started out with the new system, and half the participants started driving with the original system. This was to avoid systematic ordering effects, for example, if participants were gradually getting used to nighttime driving during the experiment. This balancing was realized by letting the first subject start with condition 1 and next subject starting with condition 2 , third subject with 1 etc.

After each of the two sessions, participants were asked to fill out a questionnaire about how they experienced the simulator and the night-vision system they have just used. In addition, participants were asked to assess their workload during the session according to the NASA-TLX scale [18].

During each session, participants were subjected to two critical situations, one where a pedestrian appeared on the side of the road, and one where a moose stood on the road. These events occurred at predetermined positions along the $140 \mathrm{~km}$ route, and were thus repeated during the second session for each participant.

There was a risk for confounds in case some of the drivers willfully decided not to brake when they detected an obstacle, if they happened to judge the obstacle to be too far away to impose any immediate danger. To avoid this risk for confounds, participants were instructed to react to any danger or obstacle they might encounter as soon as they could by pushing the sound-alarm in the middle of the steering wheel.

Participants were informed beforehand that they would be driving on a $90 \mathrm{~km}$ speed-limited highway. They were also instructed to drive as they would do normally during nighttime driving. Hence, participants were in principle free to choose a driving speed that they felt comfortable with, as long as they felt that this was appropriate on a $90 \mathrm{~km} / \mathrm{h}$ highway.

We measured participants' performance during the two sessions with respect to:
Average driving speed. Driving speed was measured during a 10-minute period relatively early in each session, before any critical events have occurred, to avoid the risk that these events affected drivers' subsequent choice of driving speed.

Distance to obstacles on the road ahead. This distance was measured at the moment when the driver reacted to the obstacle. Appearance of the critical obstacles were triggered by the own vehicle, so that they would appear at a constant distance from the vehicle. Hence, any differences in distance at the moment of reaction would reflect drivers' ability to react to danger: the longer this distance, the quicker reaction from the driver.

Eye movements were measured with a SmartEye ${ }^{\mathrm{TM}}$ eye tracker system. This system is based on two dashboard mounted IR-cameras, which feed information into a software that locates predefined characteristic points on the participant's face, and in this way determines where the participant's fovea (the black part of the eyes) are pointing.

In addition to these direct measures, the driver's mental workload was measured for each system using a NASA-TLX questionnaire [18].

Furthermore, after each of the two sessions, participants were asked to participate in a semi-structured interview, where they were asked questions about their experiences during the session, and how they liked the NVS that they had just used.

This interview was extended with additional questions about the participant's driving experience, and whether the participant judged himself/herself to be a cautious driver.

\section{B. Apparatus}

The driving simulator we used is located at Linköping University and is a fixed-base simulator, meaning that the driving cabin, called the cockpit, is mounted on a fixed platform, which cannot be skewed or tilted in sharp curves (Fig. 3). The cockpit consisted of a stripped professional truck cabin, with authentic adjustable driver seat and dashboard. SmartEye ${ }^{\mathrm{TM}}$ cameras were mounted on the dashboard, and the main instrumentation ("iron" instruments) was replaced by an exact software replica written in MacroMedia Director ${ }^{\mathrm{TM}}$.

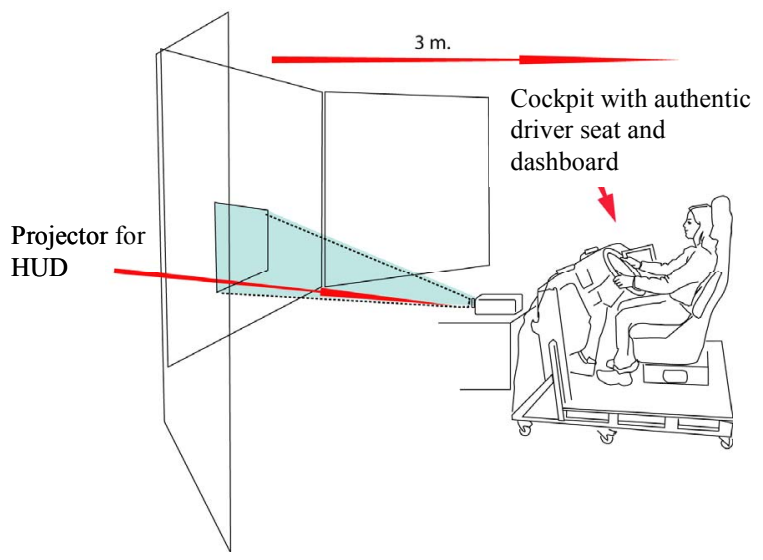

Fig. 3. The fixed-base driving simulator used in our study is softwareoriented and allows for rapid prototyping of new driver assistance systems and HMI-features. 
The simulator software, ASim, was provided by the Swedish company ACE Simulation. The architecture of ASim is module-based, which makes it possible to implement customer applications for all kinds of in-vehicle systems.

The simulated road scene was projected on three $2.4 \times 2.4 \mathrm{~m}$ screens angled in a slightly concave curve in front of the driver using three projectors. The distance between the driver and the projected road scene image was $3 \mathrm{~m}$, creating a view angle of 160 visual degrees. An additional projector was used to simulate the head-up display, which thus was overlaid the environmental presentation (see Fig. 3). This solution corresponds to projection of IR-based information onto the wind-shield in commercial vehicles.

The test route consisted of a $140 \mathrm{~km}$ long highway strip, 9 $\mathrm{m}$ wide with one lane in each direction. Participants would encounter sporadic oncoming traffic, but would not catch up with background traffic in their own direction.

Participants were subjected to two test events. In both of these, an obstacle appeared on the road at a predetermined distance from the own vehicle. In the first event, a moose appeared on the road. In the second event, a pedestrian appeared on the side of the road. As it was dark in the simulated road scene, these obstacles were not discernable by the driver at the moment of appearance, but would be detected eventually as the driver got closer to them. Because of this, the standard measure of reaction time seemed to be inadequate, as it was difficult to determine $t_{0}$, the time when the obstacle became visually discernable to the driver. Instead, we decided to use distance to the obstacle at the time the driver reacted, as a measure of drivers' ability to react promptly.

\section{RESULTS}

\section{A. Average driving speed}

The results indicate a lower average driving speed when participants were using the re-designed system. However, the difference between the two systems for all subjects was only $5 \mathrm{~km} / \mathrm{h}$, and this difference proved not to be significant above $95 \%$.

Six of the participants, however, reported in the interview that they chose the driving speed based on what they felt comfortable with, instead of looking at the speedometer. For these participants, driving speed was well above $90 \mathrm{~km} / \mathrm{h}$ (as a simulated speed of $90 \mathrm{~km} / \mathrm{h}$ felt more like $50 \mathrm{~km} / \mathrm{h}$ ), and was on the average $12 \mathrm{~km} / \mathrm{h}$ higher when these participants were using the original, continuously lit-up, display than when using the re-designed system.

\section{B. Ability to react promptly}

Due to computer failure which resulted in failure to register necessary simulator data, we only obtained distance data for the last 13 of the 23 participants. This loss of data did not change the balanced experimental design since the procedure was to change starting condition for every second subject. Data for average reaction distance did not reveal any significant differences between drivers' ability to react to obstacles using the two systems. What can be seen in the distance data is that drivers reacted to the two types of obstacles (moose and pedestrian) in an unpredictable way (revealing much variance) when they were using the original system (Fig. 4).

Drivers' reaction to obstacles, original system

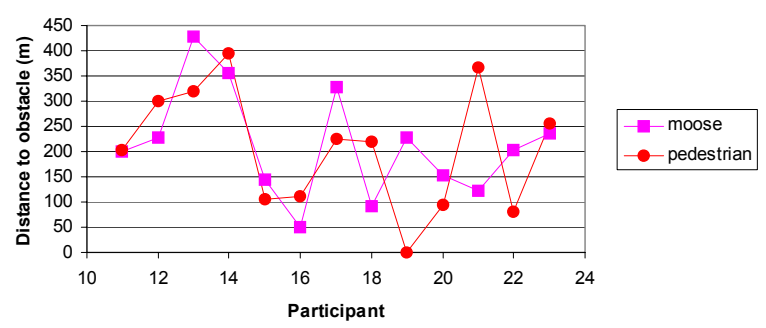

Fig. 4. Drivers' reaction to obstacles when using the original system. Drivers' reaction was measured as the distance between the vehicle and the obstacle at the moment when the driver pressed the horn on the steering wheel.

In contrast, drivers reacted in a more reliable way (showing less variance) when they were using the re-designed system (Fig. 5). Drivers' reaction was in both cases measured as the distance between the vehicle and the obstacle at the moment when the driver pressed the horn on the steering wheel. An Ftest for the equality of sample variance indicated that the spans in the two data sets are significantly different, $F(24,24)$ $=8.83, \mathrm{p}<.001$, with the range in the distance to targets at the time for detection being significantly greater in the continuous display condition.

Drivers' reaction to obstacles, re-designed system

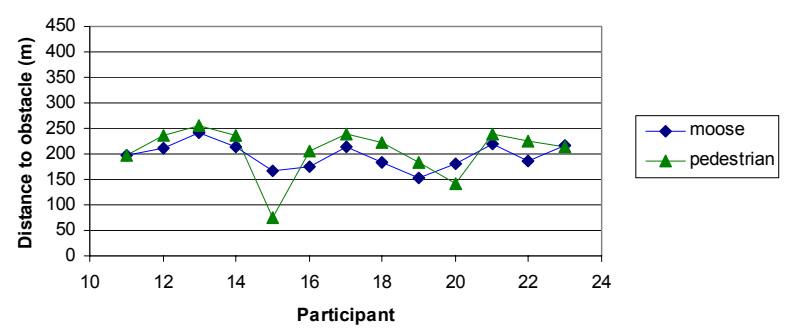

Fig. 5. Drivers' reaction to the types of obstacles (moose and pedestrian) when using the re-designed system, with unlit display during uneventful periods.

\section{Time spent looking at the display}

The SmartEye ${ }^{\mathrm{TM}}$ system that was used for measuring participants' eye movements should be individually calibrated for each participant's face. Instead, we chose to pre-calibrate the system before the experiments started using a number of "stereotypical faces" (those of the experimental leaders). This resulted in strange readings for most participants. For the few participants whose face matched well with the pre-calibrated 
ones, more reasonable data were obtained. These few data points, presumably representing a random sample of all participants, indicate that participants spent a considerable time looking at the night-vision display when they were using the original system (Table 1).

Table 1. Relative amount of time spent looking at the continuously lit head-up display for three participants.

\begin{tabular}{|c|c|}
\hline Participant & Time looking at display \\
\hline 1 & $49,5 \%$ \\
\hline 15 & $37,7 \%$ \\
\hline 22 & $74,7 \%$ \\
\hline
\end{tabular}

\section{Mental workload}

Drivers' mental workload was measured along six dimensions according to the NASA-TLX scale: mental demands, physical demands, time pressure, effort, performance, and frustration (Fig. 6). Among these, four dimensions, namely mental demands and effort revealed clear, close to significant differences, between the two systems. Mental demands turned out to be higher for the original system than for the re-designed system (68.5 vs. 46.5). Participants also experienced that they had to invest more effort into driving when using the original system as compared to the re-designed system (56.3 vs. 37.9 ).

Some differences could also be discerned in performance and frustration. Participants subjectively felt that they were performing worse when using the original system (63.1 vs. 76.3). Finally, participants felt more frustrated driving with the original than with the re-designed system (34.6 vs. 25.0).

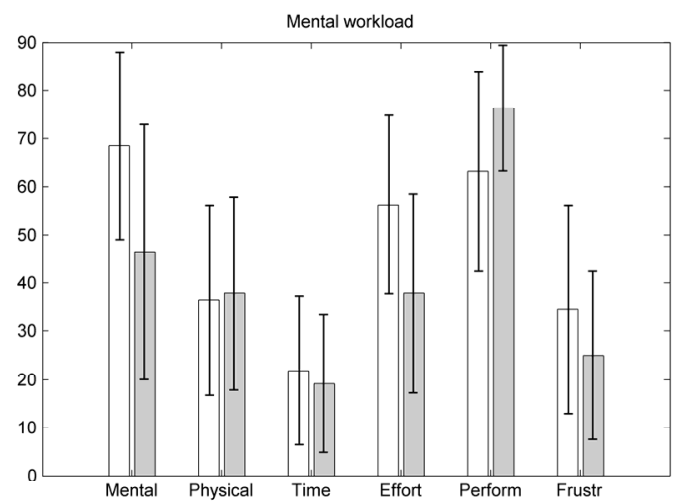

Fig. 6. Participants' subjectively experienced mental workload measured on the NASA-TLX scale. White bars show average workload for the original system. Light grey bars show average workload for the redesigned system. Vertical error bars indicate standard deviation.

\section{E. Acceptance}

Participants were asked which system they would prefer to use in real-life. All 23 participants answered that they would prefer the re-designed system with the unlit display. Many participants were disturbed by the continuously lit display of the original system, which showed not only relevant information (animate objects), but also highlighted other relatively warm objects, such as oncoming cars. Some participants said that they found it difficult to refixate between the display and the external road scene. Probably, this was due to the difference in brightness between the relatively dark road scene and the higher-contrast night-vision display, in addition to the angular and scale differences between the external scene and the displayed information.

\section{F. Driving style}

17 of the 23 participants said they thought themselves to be calm and cautious drivers. 5 participants answered that their driving style depended on if they were driving alone or if they had passengers in the car. Only 2 participants declared that they were risk takers.

\section{DISCUSSION OF RESULTS}

Driving speed was $5 \mathrm{~km} / \mathrm{h}$ higher for the original system, but this difference was not significant, presumably because participants interpreted the instructions about choice of driving speed differently. Hence, one category of participants was careful to keep the speed limit of $90 \mathrm{~km} / \mathrm{h}$, no matter what. For another category of participants, who chose a speed with which they felt comfortable with (instead of looking at the speedometer), driving speed was on the average $12 \mathrm{~km} / \mathrm{h}$ higher when using the continuously lit-up display than for the re-designed system.

That the difference is so small could in part be due to the fact that "the natural feel of speed" is limited in simulators, especially in fixed-base simulators with no feedback from road unevenness, centrifugal forces, et cetera, which are normally strong indicators of speed. In fact, a large majority of the participants reported that they felt as if they were driving at $50 \mathrm{~km} / \mathrm{h}$, when they were in fact going $90 \mathrm{~km} / \mathrm{h}$.

A second source for confound could be that participants interpreted the instructions about allowed driving speed differently. Participants were told to drive as they would normally do. They were also told that they would be driving on a $90 \mathrm{~km} / \mathrm{h}$ highway. Interpreting these instructions differently, some participants kept $90 \mathrm{~km} / \mathrm{h}$ because they were told to do so (in particular, two participants said that they did so). Others chose driving speed more freely, either based on what the speedometer showed, or based on what speed they felt comfortable with in the simulated environment.

Distance to obstacle at the moment of reaction gave significant results for difference in variance between the two systems. For the original system, detection of obstacles seems to depend on if participants happened to look at the display at the right moment or not. Variation in obstacle detection was so large for the original system that in one case the obstacle (moose) was run over! Note that such an accident would result in fatal injury to driver and passengers if it happened in reallife.

Some participants reported that with the continuous display, distant objects, such as oncoming cars looked much like pedestrians and animals (i.e., they all looked like small white dots on the display), and it was therefore difficult to 
discriminate between harmless oncoming traffic and dangerous obstacles. This may have increased the workload and decreased subjectively experienced performance levels.

High mental workload could also indicate that participants had to frequently refixate between the continuously lit headup display and the external road scene. The fact that participants also reported higher frustration because they had to constantly refixate supports this latter assumption.

Probably in response to the higher workload and experienced decreased performance, all 23 participants preferred the re-designed system.

\section{SUMMARY}

The traditional system with continuously lit night-vision display seems to have promoted a driving style where the display was constantly monitored, with short intermittent glances at the external road scene (participants spent up to $74 \%$ of their time looking at the night-vision display). This driving style seems to have two drawbacks.

First, drivers' obstacle detection becomes haphazard, with reaction times depending on whether the driver happens to look at (and attend) the display at the right moment (i.e., when the obstacle becomes distinguishable from other objects on the display).

Second, drivers who drive by feeling (not looking at the speedometer) seems to drive considerably $(12 \mathrm{~km} / \mathrm{h})$ faster, when they are provided with a continuously lit night-vision display, presumably due to improved visibility of the road ahead.

These changes in driving behavior could eliminate the safety margin created by the NVS.

In contrast, the re-designed system with a night vision display that was unlit during uneventful periods (i.e. when there were no obstacles ahead) promoted lower speeds and more reliable obstacle detection (with less variance in reaction times). In this case, the system chose the right moment when the display should be monitored and warned the driver by lighting up the display. Accordingly, the re-designed system was reported to be less mentally loading, and was preferred by all 23 participants.

A concluding comment is that this study did not include any optimization of the display presentation for the new system. Raw sensor information may not be the best alternative. Also questions on when or how to use this display resource for other purposes during uneventful periods remain to be investigated. However, we strongly believe that the introduction of HUDs in cars could have a positive impact on driving safety, when designed carefully.

\section{ACKNOWLEDGEMENTS}

We would like to thank the following students: Ingrid Alin Nilsson, Helen Alm, Sona Arabloui, Henrik Gemoll, Anna Heyden, Karl-Johan Törngren, and Rasmus Wetterström, who conducted the experiment, under the supervision of Torbjörn Alm and Rita Kovordányi. We are also much obliged to Calle
Isaksson at ACE Simulation ${ }^{\mathrm{TM}}$, who helped with scenario programming, and Vitalij Savin, for managing the simulator facilities. Finally, we would like to thank Scania AB, Södertälje, Sweden, for sponsoring the project.

\section{REFERENCES}

[1] Z. Jeftic, J. Engström, and P. Piamonte, "Potential safety benefits of lane departure warning systems on Swedish roads: Pre-study", Swedish National Road Agency, Publication 2003:14, ISBN: 1401-9612, 2003.

[2] R. Kovordányi, K. Ohlsson, \& T. Alm, "Dynamically deployed support as a potential solution to negative behavioral adaptation". In Proc. of 2005 IEEE Intelligent Vehicles Symposium, 2005.

[3] C. M. Brown, "The concept of behavioural adaptation: Does it occur in response to lane departure warnings?", in Proc. Intl. Conf. Traffic and Transport Psychology, pp. 4-7, 2000.

[4] C. M. Rudin-Brown and Y. I. Noy, "Investigation of behavioral adaptation to lane departure warnings", Transportation Research record, vol. 1803 , pp. $30-37,2002$.

[5] C. M. Rudin-Brown and H. A. Parker, "Behavioral adaptation to adaptive cruise control (ACC): implications for preventive strategies", Transportation Research, part F, vol. 7, 2004, pp. 59-76.

[6] P. C. Burns, "Behavioural Adaptation to an Advanced Driver Support System", Volvo Technology Corporation, Internal Report, 2001.

[7] OECD, "Behavioural adaptation to changes in the road transport systems", OECD, Paris, 1990.

[8] H. Summala, "Behavioural adaptation and drivers' task control", in Human factors for highway engineers, R. Fuller and J. A. Santos, Eds. Oxford: Pergamon Press, 2002.

[9] Carpages, "Honda's intelligent night vision system in four steps". http://www.carpages.co.uk/honda/honda intelligent_night_vision_syste m $26 \quad 10$ 04.asp? switched $=$ on\&echo $=977170796 /$ Visited on 2005-12-15.

[10] HONDA, "Honda's latest intelligent night vision system". http://world.honda.com/HDTV/IntelligentNightVision/200408/ visited on 2005-12-15.

[11] G. J. S. Wilde, "The theory of risk homeostatis: Implications for traffic safety and health", Risk Analysis, vol. 2, 1982, pp. 209-225.

[12] H. Summala and R. Näätänen, "The zero-risk theory and overtaking decisions," in Road user behavior: Theory and research, $\mathrm{T}$. Rothengatter and R. de Bruin, Eds. Van Gorcum, Assen / Maastricht. 1988, pp. 82-92.

[13] H. Summala, "Hierarchical model of behavioural adaptation and traffic accidents", in Traffic and Transport Psychology: Theory and application, T. Rothengatter and E. C. Vaya, Eds. Oxford: Pergamon Press, 1997, pp. 41-52.

[14] P. C. Cacciabue, "Modelling and simulation of human behaviour in system control”. London: Springer Verlag, 1998.

[15] W. van Winsum, "From adaptive control to adaptive behaviour." $\mathrm{PhD}$ thesis, University of Groningen, Netherlands, 1996.

[16] A. Smiley, "Behavioural adaptation, safety, and intelligent transportation systems”. Transportation Research Record, vol. 724, 47-51, 2000.

[17] T. Alm, K. Ohlsson, \& R. Kovordányi, "Glass Cockpit Simulators Tools for IT-based Car Systems Design \& Evaluation”. In Proc. of the Driving Simulator Conference - North America, 2005.

[18] S. Hart and L. E. Staveland, "Development of NASA-TLX (Task Load Index): results of empirical and theoretical research", in P. A. Hancock and N. Meshkati, Eds. Human Mental Workload. Amsterdam: Elsevier Science Publishers, 1988. 